AperTO - Archivio Istituzionale Open Access dell'Università di Torino

\title{
Some Remarks on Connected Coalgebras
}

\section{This is the author's manuscript}

Original Citation:

Availability:

This version is available http://hdl.handle.net/2318/92961

since

Published version:

DOI:10.1007/s10468-009-9147-4

Terms of use:

Open Access

Anyone can freely access the full text of works made available as "Open Access". Works made available under a Creative Commons license can be used according to the terms and conditions of said license. Use of all other works requires consent of the right holder (author or publisher) if not exempted from copyright protection by the applicable law. 


\title{
(9.) \\ UNIVERSITÀ DEGLI STUDI DI TORINO
}

This is an author version of the contribution published on:

\begin{abstract}
Alessandro ARDIZZONI and Claudia MENINI. Some Remarks on Connected Coalgebras. Algebras and Representation Theory, October 2009, Volume 12, Issue 2-5, pp 235-249. DOI: $10.1007 / \mathrm{s} 10468-009-9147-4$
\end{abstract}

The definitive version is available at:

http://link.springer.com/article/10.1007\%2Fs10468-009-9147-4 


\title{
SOME REMARKS ON CONNECTED COALGEBRAS
}

\author{
A. ARDIZZONI AND C. MENINI
}

Dedicated to Freddy Van Oystaeyen, on the occasion of his sixtieth birthday

\begin{abstract}
In this paper we introduce the notions of connected, 0-connected and strictly graded coalgebra in the framework of an abelian monoidal category $\mathcal{M}$ and we investigate the relations between these concepts. We recover several results, involving these notions, which are well known in the case when $\mathcal{M}$ is the category of vector spaces over a field $K$. In particular we characterize when a 0-connected graded bialgebra is a bialgebra of type one.
\end{abstract}

\section{INTRODUCTION}

Let $\mathcal{M}$ be a coabelian monoidal category such that the tensor product commutes with direct sums. Given a graded coalgebra $\left(C=\oplus_{n \in \mathbb{N}} C_{n}, \Delta, \varepsilon\right)$ in $\mathcal{M}$, we can write $\Delta_{\mid C_{n}}$ as the sum of unique components $\Delta_{i, j}: C_{i+j} \rightarrow C_{i} \otimes C_{j}$ where $i+j=n$. The coalgebra $C$ is defined to be a strongly $\mathbb{N}$-graded coalgebra (see [AM], Definition 2.9]) when $\Delta_{i, j}^{C}: C_{i+j} \rightarrow C_{i} \otimes C_{j}$ is a monomorphism for every $i, j \in \mathbb{N}$. The associated graded coalgebra

$$
g r_{C} E=C \oplus \frac{C \wedge_{E} C}{C} \oplus \frac{C \wedge_{E} C \wedge_{E} C}{C \wedge_{E} C} \oplus \cdots,
$$

for a given subcoalgebra $C$ of a coalgebra $E$ in $\mathcal{M}$, is an example of strongly $\mathbb{N}$-graded coalgebra (see [AM2, Theorem 2.10]).

A graded coalgebra $\left(C=\oplus_{n \in \mathbb{N}} C_{n}, \Delta_{C}, \varepsilon_{C}\right)$ in a cocomplete monoidal category $\mathcal{M}$ is called 0-connected whenever $\varepsilon_{C} i_{0}^{C}: C_{0} \rightarrow \mathbf{1}$ is an isomorphism where $i_{0}^{C}: C_{0} \rightarrow C$ denotes the canonical injection. $C$ is called strictly graded whenever it is both strongly $\mathbb{N}$-graded and 0 -connected. The associated graded coalgebra $g r_{1} C$ of a coaugmented coalgebra $C$ in $\mathcal{M}$ is an example of a strictly graded coalgebra (see Theorem $[\mathbf{L})$. We also introduce the notion of connected coalgebra in $\mathcal{M}$ (see Definition [2.]).

In Theorem $\left[\mathbf{D}\right.$ we prove the following result. Let $\left(C=\oplus_{n \in \mathbb{N}} C_{n}, \Delta_{C}, \varepsilon_{C}\right)$ be a 0-connected graded coalgebra in a cocomplete coabelian monoidal category $\mathcal{M}$. Then

1) $\left(\left(C, \Delta_{C}, \varepsilon_{C}\right), u_{C}=i_{\mathbf{1}}^{C}\right)$ is a connected coalgebra where $u_{C}:=i_{0}^{C} \varepsilon_{0}^{-1}: \mathbf{1} \rightarrow C$;

2) $C_{0} \wedge_{C} C_{0}=C_{0} \oplus P(C)$, where $P(C)$ denotes the primitive part of $C$.

Moreover, if $\mathcal{M}$ is also complete and satisfies $A B 5$, the following assertions are equivalent:

(a) $C$ is a strongly $\mathbb{N}$-graded coalgebra;

(b) $C_{1}=P(C)$.

This result is then applied to the following setting. Let $H$ be a braided bialgebra in a cocomplete and complete abelian braided monoidal category $(\mathcal{M}, c)$ satisfying $A B 5$. Assume that the tensor product commutes with direct sums and is two-sided exact. Let $M$ be in ${ }_{H}^{H} \mathcal{M}_{H}^{H}$. Let $T=T_{H}(M)$ be the relative tensor algebra and let $T^{c}=T_{H}^{c}(M)$ be the relative cotensor coalgebra as introduced in [AMS]. In [AM], we proved that both $T$ and $T^{c}$ have a natural structure of graded braided bialgebra and that the natural algebra morphism from $T$ to $T^{c}$, which coincides with the canonical injections on $H$ and $M$, is a graded bialgebra homomorphism. Thus its image is a graded braided

1991 Mathematics Subject Classification. Primary 18D10; Secondary 16W30.

Key words and phrases. Monoidal Categories, Connected Coalgebras, Graded Coalgebras, Braided Bialgebras.

This paper was written while the authors were members of G.N.S.A.G.A. with partial financial support from Mi.U.R.. 
bialgebra which we denote by $H[M]$ and call, accordingly to $[\mathbb{\nabla i}]$, the braided bialgebra of type one associated to $H$ and $M$ (see [AM], Definition 6.7]).

Let now $\left(B, m_{B}, u_{B}, \Delta_{B}, \varepsilon_{B}\right)$ be a braided graded bialgebra in $(\mathcal{M}, c)$. Assume that $B$ is 0 connected as a coalgebra. Then, by the foregoing, $\left(\left(B, \Delta_{B}, \varepsilon_{B}\right), u_{B}\right)$ is a connected coalgebra. We prove (see Theorem [2]3) that $B$ is the braided bialgebra of type one $B_{0}\left[B_{1}\right]$ associated to $B_{0}$ and $B_{1}$ if and only if

$$
\left(\oplus_{n \geq 1} B_{n}\right)^{2}=\oplus_{n \geq 2} B_{n} \quad \text { and } \quad P(B)=B_{1} .
$$

Therefore TOBAs, as introduced in [AG], Definition 3.2.3], are exactly the braided bialgebras of type one in $\mathcal{M}={ }_{H}^{H} \mathcal{Y D}$ which are 0 -connected.

\section{Preliminaries and Notations}

Notations. Let $\left[\left(X, i_{X}\right)\right]$ be a subobject of an object $E$ in an abelian category $\mathcal{M}$, where $i_{X}=i_{X}^{E}: X \hookrightarrow E$ is a monomorphism and $\left[\left(X, i_{X}\right)\right]$ is the associated equivalence class. By abuse of language, we will say that $\left(X, i_{X}\right)$ is a subobject of $E$ and we will write $\left(X, i_{X}\right)=\left(Y, i_{Y}\right)$ to mean that $\left(Y, i_{Y}\right) \in\left[\left(X, i_{X}\right)\right]$. The same convention applies to cokernels. If $\left(X, i_{X}\right)$ is a subobject of $E$ then we will write $\left(E / X, p_{X}\right)=\operatorname{Coker}\left(i_{X}\right)$, where $p_{X}=p_{X}^{E}: E \rightarrow E / X$.

Let $\left(X_{1}, i_{X_{1}}^{Y_{1}}\right)$ be a subobject of $Y_{1}$ and let $\left(X_{2}, i_{X_{2}}^{Y_{2}}\right)$ be a subobject of $Y_{2}$. Let $x: X_{1} \rightarrow X_{2}$ and $y: Y_{1} \rightarrow Y_{2}$ be morphisms such that $y \circ i_{X_{1}}^{Y_{1}}=i_{X_{2}}^{Y_{2}} \circ x$. Then there exists a unique morphism, which we denote by $y / x=\frac{y}{x}: Y_{1} / X_{1} \rightarrow Y_{2} / X_{2}$, such that $\frac{y}{x} \circ p_{X_{1}}^{Y_{1}}=p_{X_{2}}^{Y_{2}} \circ y$ :

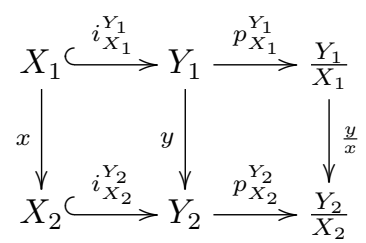

$\delta_{u, v}$ will denote the Kronecker symbol for every $u, v \in \mathbb{N}$.

1.1. Monoidal Categories. Recall that (see [Ka, Chap. XI]) a monoidal category is a category $\mathcal{M}$ endowed with an object $\mathbf{1} \in \mathcal{M}$ (called unit), a functor $\otimes: \mathcal{M} \times \mathcal{M} \rightarrow \mathcal{M}$ (called tensor product), and functorial isomorphisms $a_{X, Y, Z}:(X \otimes Y) \otimes Z \rightarrow X \otimes(Y \otimes Z), l_{X}: \mathbf{1} \otimes X \rightarrow X$, $r_{X}: X \otimes \mathbf{1} \rightarrow X$, for every $X, Y, Z$ in $\mathcal{M}$. The functorial morphism $a$ is called the associativity constraint and satisfies the Pentagon Axiom, that is the following relation

$$
\left(U \otimes a_{V, W, X}\right) \circ a_{U, V \otimes W, X} \circ\left(a_{U, V, W} \otimes X\right)=a_{U, V, W \otimes X} \circ a_{U \otimes V, W, X}
$$

holds true, for every $U, V, W, X$ in $\mathcal{M}$. The morphisms $l$ and $r$ are called the unit constraints and they obey the Triangle Axiom, that is $\left(V \otimes l_{W}\right) \circ a_{V, \mathbf{1}, W}=r_{V} \otimes W$, for every $V, W$ in $\mathcal{M}$.

A braided monoidal category $(\mathcal{M}, c)$ is a monoidal category $(\mathcal{M}, \otimes, \mathbf{1})$ equipped with a braiding $c$, that is a natural isomorphism $c_{X, Y}: X \otimes Y \longrightarrow Y \otimes X$ for every $X, Y, Z$ in $\mathcal{M}$ satisfying

$$
c_{X \otimes Y, Z}=\left(c_{X, Z} \otimes Y\right)\left(X \otimes c_{Y, Z}\right) \quad \text { and } \quad c_{X, Y \otimes Z}=\left(Y \otimes c_{X, Z}\right)\left(c_{X, Y} \otimes Z\right) .
$$

For further details on these topics, we refer to [Ka, Chapter XIII].

It is well known that the Pentagon Axiom completely solves the consistency problem arising out of the possibility of going from $((U \otimes V) \otimes W) \otimes X$ to $U \otimes(V \otimes(W \otimes X))$ in two different ways (see [M]1, page 420]). This allows the notation $X_{1} \otimes \cdots \otimes X_{n}$ forgetting the brackets for any object obtained from $X_{1}, \cdots X_{n}$ using $\otimes$. Also, as a consequence of the coherence theorem, the constraints take care of themselves and can then be omitted in any computation involving morphisms in a monoidal category $\mathcal{M}$.

Thus, for sake of simplicity, from now on, we will omit the associativity constraints.

The notions of algebra, module over an algebra, coalgebra and comodule over a coalgebra can be introduced in the general setting of monoidal categories. Given an algebra $A$ in $\mathcal{M}$ on can define the categories ${ }_{A} \mathcal{M}, \mathcal{M}_{A}$ and ${ }_{A} \mathcal{M}_{A}$ of left, right and two-sided modules over $A$ respectively. Similarly, 
given a coalgebra $C$ in $\mathcal{M}$, one can define the categories of $C$-comodules ${ }^{C} \mathcal{M}, \mathcal{M}^{C},{ }^{C} \mathcal{M}^{C}$. For more details, the reader is referred to [AMS2].

Definitions 1.2 . Let $\mathcal{M}$ be a monoidal category.

We say that $\mathcal{M}$ is a coabelian monoidal category if $\mathcal{M}$ is abelian and both the functors $X \otimes(-): \mathcal{M} \rightarrow \mathcal{M}$ and $(-) \otimes X: \mathcal{M} \rightarrow \mathcal{M}$ are additive and left exact, for any $X \in \mathcal{M}$.

1.3. Let $\mathcal{M}$ be a coabelian monoidal category.

Let $\left(C, i_{C}^{E}\right)$ and $\left(D, i_{D}^{E}\right)$ be two subobjects of a coalgebra $(E, \Delta, \varepsilon)$. Set

$$
\begin{gathered}
\Delta_{C, D}:=\left(p_{C}^{E} \otimes p_{D}^{E}\right) \Delta: E \rightarrow \frac{E}{C} \otimes \frac{E}{D} \\
\left(C \wedge_{E} D, i_{C \wedge_{E} D}^{E}\right)=\operatorname{ker}\left(\Delta_{C, D}\right), \quad i_{C \wedge_{E} D}^{E}: C \wedge_{E} D \rightarrow E \\
\left(\frac{E}{C \wedge_{E} D}, p_{C \wedge_{E} D}^{E}\right)=\operatorname{Coker}\left(i_{C \wedge_{E} D}^{E}\right)=\operatorname{Im}\left(\Delta_{C, D}\right), \quad p_{C \wedge_{E} D}^{E}: E \rightarrow \frac{E}{C \wedge_{E} D}
\end{gathered}
$$

Moreover, we have the following exact sequence:

$$
0 \longrightarrow C \wedge_{E} D \stackrel{i_{C \wedge_{E} D}^{E}}{\longrightarrow} E \stackrel{p_{C \wedge_{E} D}^{E}}{\longrightarrow} \frac{E}{C \wedge_{E} D} \longrightarrow 0 .
$$

Assume now that $\left(C, i_{C}^{E}\right)$ and $\left(D, i_{D}^{E}\right)$ are two subcoalgebras of $(E, \Delta, \varepsilon)$. Since $\Delta_{C, D} \in{ }^{E} \mathcal{M}^{E}$, it is straightforward to prove that $C \wedge_{E} D$ is a coalgebra and that $i_{C \wedge_{E} D}^{E}$ is a coalgebra homomorphism.

Let $\left(C, i_{C}^{E}\right)$ be a subobject of a coalgebra $(E, \Delta, \varepsilon)$ in a coabelian monoidal category $\mathcal{M}$. We can define (see [AMS2] ) the $n$-th wedge product $\left(C^{\wedge_{E} n}, i_{C^{\wedge} E^{n}}^{E}\right)$ of $C$ in $E$ where $i_{C^{\wedge} E^{n}}^{E}: C^{\wedge_{E} n} \rightarrow E$. By definition, we have

$$
C^{\wedge_{E} 0}=0 \quad \text { and } \quad C^{\wedge_{E} n}=C^{\wedge_{E} n-1} \wedge_{E} C, \text { for every } n \geq 1 .
$$

One can check that $C^{\wedge_{E} i} \wedge_{E} C^{\wedge_{E} j}=C^{\wedge_{E} i+j}$ for every $i, j \in \mathbb{N}$.

Assume now that $\left(C, i_{C}^{E}\right)$ is a subcoalgebra of the coalgebra $(E, \Delta, \varepsilon)$. Then there is a (unique) coalgebra homomorphism

$$
i_{C^{\wedge} E^{n}}^{C^{\wedge^{n+1}}}: C^{\wedge{ }_{E} n} \rightarrow C^{\wedge_{E} n+1} \text {, for every } n \in \mathbb{N} .
$$

such that $i_{C^{\wedge_{E}}{ }^{n+1}}^{{ }^{\prime}} \circ i_{C^{\wedge_{E}}}^{{C^{n}}^{n+1}}=i_{C^{\wedge} E^{n}}^{E}$.

1.4. Graded Objects. Let $\left(X_{n}\right)_{n \in \mathbb{N}}$ be a sequence of objects in a cocomplete coabelian monoidal category $\mathcal{M}$ and let

$$
X=\bigoplus_{n \in \mathbb{N}} X_{n}
$$

be their coproduct in $\mathcal{M}$. In this case we also say that $X$ is a graded object of $\mathcal{M}$ and that the sequence $\left(X_{n}\right)_{n \in \mathbb{N}}$ defines a grading on $X$. A morphism

$$
f: X=\bigoplus_{n \in \mathbb{N}} X_{n} \rightarrow Y=\bigoplus_{n \in \mathbb{N}} Y_{n}
$$

is called a graded homomorphism whenever there exists a family of morphisms $\left(f_{n}: X_{n} \rightarrow Y_{n}\right)_{n \in \mathbb{N}}$ such that $f=\oplus_{n \in \mathbb{N}} f_{n}$ i.e. such that

$$
f \circ i_{X_{n}}^{X}=i_{Y_{n}}^{Y} \circ f_{n} \text {, for every } n \in \mathbb{N} \text {. }
$$

We fix the following notations. Throughout let

$$
p_{n}^{X}: X \rightarrow X_{n} \quad \text { and } \quad i_{n}^{X}: X_{n} \rightarrow X
$$

be the canonical projection and injection respectively, for any $n \in \mathbb{N}$.

Given graded objects $X, Y$ in $\mathcal{M}$ we set

$$
(X \otimes Y)_{n}=\oplus_{a+b=n}\left(X_{a} \otimes Y_{b}\right) .
$$

Then this defines a grading on $X \otimes Y$ whenever the tensor product commutes with direct sums. 
1.5. Let $\mathcal{M}$ be a coabelian monoidal category such that the tensor product commutes with direct sums.

Recall that a graded coalgebra in $\mathcal{M}$ is a coalgebra $(C, \Delta, \varepsilon)$ where

$$
C=\oplus_{n \in \mathbb{N}} C_{n}
$$

is a graded object of $\mathcal{M}$ such that $\Delta: C \rightarrow C \otimes C$ is a graded homomorphism i.e. there exists a family $\left(\Delta_{n}\right)_{n \in \mathbb{N}}$ of morphisms

$$
\Delta_{n}^{C}=\Delta_{n}: C_{n} \rightarrow(C \otimes C)_{n}=\oplus_{a+b=n}\left(C_{a} \otimes C_{b}\right) \text { such that } \Delta=\oplus_{n \in \mathbb{N}} \Delta_{n} .
$$

We set

$$
\Delta_{a, b}^{C}=\Delta_{a, b}:=\left(C_{a+b} \stackrel{\Delta_{a+b}}{\rightarrow}(C \otimes C)_{a+b} \stackrel{\omega_{a, b}^{C, C}}{\rightarrow} C_{a} \otimes C_{b}\right) .
$$

A homomorphism $f:\left(C, \Delta_{C}, \varepsilon_{C}\right) \rightarrow\left(D, \Delta_{D}, \varepsilon_{D}\right)$ of coalgebras is a graded coalgebra homomorphism if it is a graded homomorphism too.

Definition 1.6. [AM], Definition 2.9] Let $\left(C=\oplus_{n \in \mathbb{N}} C_{n}, \Delta, \varepsilon\right)$ be a graded coalgebra in $\mathcal{M}$. In analogy with the group graded case (see $[\mathbb{N} \mathbb{W}]$ ), we say that $C$ is a strongly $\mathbb{N}$-graded coalgebra whenever

$$
\Delta_{i, j}^{C}: C_{i+j} \rightarrow C_{i} \otimes C_{j} \text { is a monomorphism for every } i, j \in \mathbb{N},
$$

where $\Delta_{i, j}^{C}$ is the morphism defined in Definition $\square$.

\section{Connected coalgebras}

Definitions 2.1. Let $\mathcal{M}$ be a coabelian monoidal category. A coaugmented coalgebra $((C, \Delta, \varepsilon), u)$ in $\mathcal{M}$ consists of a coalgebra $(C, \Delta, \varepsilon)$ endowed with a coalgebra homomorphism $u: \mathbf{1} \rightarrow C$ called coaugmentation of $C$. Note that $u$ is a monomorphism as $\varepsilon u=\mathrm{Id}_{\mathbf{1}}$. Given a coaugmented coalgebra $((C, \Delta, \varepsilon), u)$ define

$$
\begin{gathered}
\alpha_{C}:=\left(C \otimes u_{C}\right) \circ r_{C}^{-1}+\left(u_{C} \otimes C\right) \circ l_{C}^{-1}-\Delta_{C}: C \rightarrow C \otimes C, \\
\left(P(C), i_{P(C)}\right)=\operatorname{ker}\left(\alpha_{C}\right) .
\end{gathered}
$$

$\left(P(C), i_{P(C)}\right)$ is called the primitive part of the coaugmented coalgebra $C$.

A connected coalgebra in $\mathcal{M}$ is a coaugmented coalgebra $((C, \Delta, \varepsilon), u)$ in $\mathcal{M}$ such that

$$
\left.\underset{\lim _{\longrightarrow}}{\left(\mathbf{1}^{\wedge}{ }_{C}^{n}\right.}\right)_{n \in \mathbb{N}}=C .
$$

REMARK 2.2. Let $\mathcal{M}$ be the category of vector spaces over a field $K$ and let $((C, \Delta, \varepsilon), u)$ be a connected coalgebra in $\mathcal{M}$ accordingly to the previous definition. Then $C_{(0)}:=\operatorname{Corad}(C) \subseteq \operatorname{Im}(u)$ (see e.g. [AMSD, Lemma 5.2]) and hence $C_{(0)}=\operatorname{Im}(u)$ so that $C$ is connected in the usual sense. On the other hand, since $C=\underset{\lim }{\longrightarrow}\left(C_{(0)}^{\wedge_{C}^{n}}\right)_{n \in \mathbb{N}}$ it is clear that an ordinary connected coalgebra $C$ is also a connected coalgebra in $\overrightarrow{\mathcal{M}}$.

REMARK 2.3. Let $C$ be a connected coalgebra in the monoidal category of vector spaces over a field $K$. Then, the cotensor coalgebra $T^{c}=T_{C}^{c}(M)$ is strongly $\mathbb{N}$-graded and connected for every $C$-bicomodule $M$. Nevertheless $C$ needs not to be $K$, in general.

Question 2.4. Let $\mathcal{M}$ be a cocomplete coabelian monoidal category and let $((C, \Delta, \varepsilon), u)$ be a connected coalgebra in $\mathcal{M}$. Let $M$ be a $C$-bicomodule in $\mathcal{M}$. Is it true that the cotensor coalgebra $T_{C}^{c}(M)$ is a connected coalgebra?

Lemma 2.5. Let $\left(C, u_{C}\right)$ be a coaugmented coalgebra and let $f: C \rightarrow D$ be a coalgebra homomorphism in a coabelian monoidal category $\mathcal{M}$. Then $\left(D, u_{D}\right)$ is a coaugmented coalgebra where $u_{D}=f \circ u_{C}$. Moreover

$$
\alpha_{D} \circ f=(f \otimes f) \circ \alpha_{C} .
$$


Proof. Clearly $\left(D, u_{D}\right)$ is a coaugmented coalgebra. Moreover, we have

$$
\begin{aligned}
\alpha_{D} \circ f & =\left[\left(D \otimes u_{D}\right) \circ r_{D}^{-1}+\left(u_{D} \otimes D\right) \circ l_{D}^{-1}-\Delta_{D}\right] \circ f \\
& =\left(D \otimes f \circ u_{C}\right) \circ r_{D}^{-1} \circ f+\left(f \circ u_{C} \otimes D\right) \circ l_{D}^{-1} \circ f-\Delta_{D} \circ f \\
& =\left(D \otimes f \circ u_{C}\right) \circ(f \otimes \mathbf{1}) \circ r_{C}^{-1}+\left(f \circ u_{C} \otimes D\right) \circ(\mathbf{1} \otimes f) \circ l_{C}^{-1}-(f \otimes f) \circ \Delta_{C} \\
& =(f \otimes f) \circ\left[\left(C \otimes u_{C}\right) \circ r_{C}^{-1}+\left(u_{C} \otimes C\right) \circ l_{C}^{-1}-\Delta_{C}\right]=(f \otimes f) \circ \alpha_{C} .
\end{aligned}
$$

Lemma 2.6. Let $i_{F}^{E}: F \rightarrow E$ and $i_{G}^{E}: G \rightarrow E$ be monomorphisms which are coalgebra homomorphisms in a coabelian monoidal category $\mathcal{M}$. Then

$$
\left(\frac{F \wedge_{E} G}{G},{ }^{F} \rho_{\frac{F \wedge_{E} G}{G}}: \frac{F \wedge_{E} G}{G} \rightarrow F \otimes \frac{F \wedge_{E} G}{G}\right)
$$

is a left $F$-comodule where $\frac{F}{\rho_{\frac{F \wedge_{E} G}{G}}}$ is uniquely defined by

$$
{ }^{E} \rho_{\frac{F \wedge_{E} G}{G}}=\left(i_{F}^{E} \otimes \frac{F \wedge_{E} G}{G}\right) \circ{ }^{F} \rho_{\frac{F \wedge_{E} G}{G}} .
$$

Furthermore the following diagram

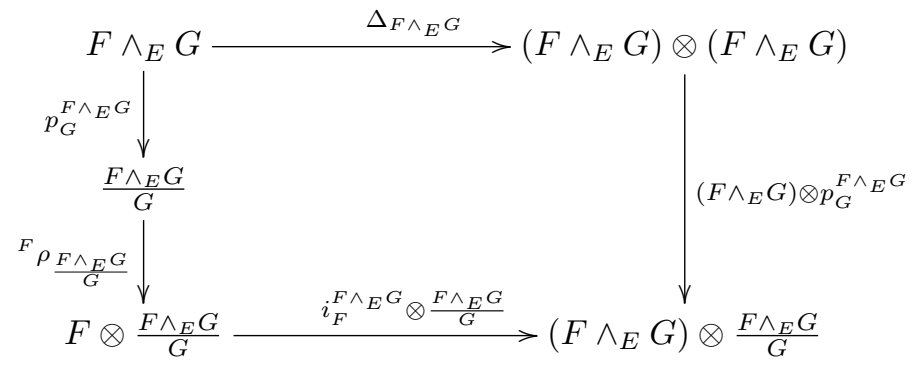

is commutative and

$$
{ }^{1} \rho_{\frac{1 \wedge_{E} G}{G}}=l^{-1} \frac{1 \wedge_{E} G}{G}
$$

whenever $F=\mathbf{1}$.

Proof. The first part of the statement follows by [A], Lemma 2.14].

Let us prove the commutativity of the diagram. We have

$$
\begin{aligned}
& \left(i_{F \wedge_{E} G}^{E} \otimes \frac{F \wedge_{E} G}{G}\right) \circ\left(i_{F}^{F \wedge_{E} G} \otimes \frac{F \wedge_{E} G}{G}\right) \circ{ }^{F} \rho_{\frac{F \wedge_{E} G}{G}} \circ p_{G}^{F \wedge G} \\
= & \left(i_{F}^{E} \otimes \frac{F \wedge_{E} G}{G}\right) \circ{ }^{F} \rho_{\frac{F \wedge_{E} G}{G}} \circ p_{G}^{F \wedge_{E} G}={ }^{E} \rho_{\frac{F \wedge_{E} G}{G}} \circ p_{G}^{F \wedge_{E} G} \\
= & \left(E \otimes p_{G}^{F \wedge_{E} G}\right) \circ{ }^{E} \rho_{F \wedge_{E} G}=\left(i_{F \wedge_{E} G}^{E} \otimes p_{G}^{F \wedge_{E} G}\right) \circ \Delta_{F \wedge_{E} G} .
\end{aligned}
$$

Since the tensor product is left exact, then $i_{F \wedge_{E} G}^{E} \otimes \frac{F \wedge_{E} G}{G}$ is a monomorphism so that we obtain the commutativity of the diagram. Finally, since

$$
l^{-1}{ }_{\frac{F \wedge_{E} G}{G}}=\left(\varepsilon_{F} \otimes \frac{F \wedge_{E} G}{G}\right) \circ{ }^{F} \rho_{\frac{F \wedge_{E} G}{G}} \quad \text { and } \quad \varepsilon_{\mathbf{1}}=\operatorname{Id}_{\mathbf{1}}
$$

when $F=\mathbf{1}$ we obtain the last equality in the statement.

Lemma 2.7. Let $\left(E, u_{E}=i_{1}^{E}\right)$ be a coaugmented coalgebra in a coabelian monoidal category $\mathcal{M}$. Then

$$
\left(\mathbf{1}^{\wedge_{E}^{n}}, u_{\mathbf{1}^{\wedge}{ }_{E}^{n}}=i_{\mathbf{1}}^{\wedge^{\wedge} E}\right)
$$


is a coaugmented coalgebra for every $n \in \mathbb{N}$. Furthermore, for every $n \in \mathbb{N}$, there exists a unique morphism $\tau_{n}: \mathbf{1}^{\wedge_{E}^{n+1}} \rightarrow \mathbf{1}^{\wedge_{E}^{n}} \otimes \mathbf{1}^{\wedge_{E}^{n}}$ such that the following diagram

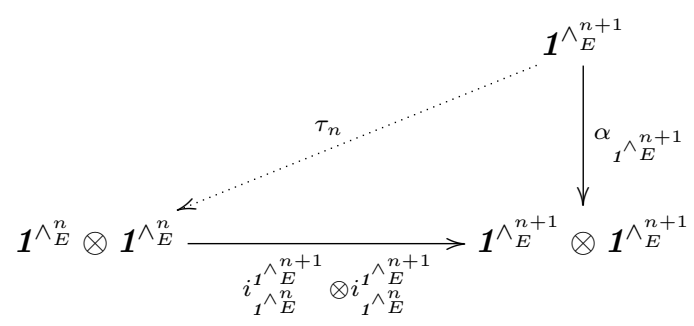

is commutative.

Proof. Set $\mathbf{1}^{n}:=\mathbf{1}^{\wedge}{ }_{E}^{n}$, for every $n \in \mathbb{N}$.

Since $\left(\mathbf{1}, u_{\mathbf{1}}=\operatorname{Id}_{\mathbf{1}}\right)$ is a coaugmented coalgebra and $i_{\mathbf{1}^{1}}^{\mathbf{1}^{n}}$ is a coalgebra homomorphism, in view of Lemma [2.5, it is clear that $\left(\mathbf{1}^{\wedge_{E}^{n}}, u_{\mathbf{1}^{\wedge}{ }_{E}^{n}}=i_{\mathbf{1}^{1}}^{\mathbf{1}^{n}}\right)$ is also a coaugmented coalgebra.

Consider the following exact sequence

$$
0 \longrightarrow \mathbf{1}^{n} \stackrel{i_{\mathbf{1}^{n}}^{\mathbf{n}^{n+1}}}{\longrightarrow} \mathbf{1}^{n+1} \stackrel{p_{\mathbf{1}^{n}}^{\mathbf{n}^{n+1}}}{\longrightarrow} \frac{\mathbf{1}^{n+1}}{\mathbf{1}^{n}} \longrightarrow 0
$$

were $p_{\mathbf{1}^{n}}^{\mathbf{1}^{n+1}}$ denotes the canonical projection. By applying the functor $\mathbf{1}^{n+1} \otimes(-)$ we get

$$
0 \longrightarrow \mathbf{1}^{n+1} \otimes \mathbf{1}^{n} \stackrel{\mathbf{1}^{n+1} \otimes i_{\mathbf{1}^{n}}^{\mathbf{n}^{n+1}}}{\beta_{n} \cdots \cdots \cdots \cdots \cdots \cdots} \mathbf{1}^{n+1} \underset{\mathbf{1}^{n+1}}{\otimes \alpha_{\mathbf{1}^{n}}} \mathbf{1}^{n+1} \stackrel{\mathbf{1}^{n+1} \otimes p_{\mathbf{1}^{1^{n+1}}}}{\longrightarrow} \mathbf{1}^{n+1} \otimes \frac{\mathbf{1}^{n+1}}{\mathbf{1}^{n}}
$$

By Lemma [2.6], we have

$$
\left(i_{\mathbf{1}}^{\mathbf{1} \wedge_{E} \mathbf{1}^{n}} \otimes \frac{\mathbf{1} \wedge_{E} \mathbf{1}^{n}}{\mathbf{1}^{n}}\right) \circ{ }^{\mathbf{1}} \rho_{\frac{1 \wedge_{E} \mathbf{1}^{n}}{\mathbf{1}^{n}}} \circ p_{\mathbf{1}^{n}}^{\mathbf{1} \wedge_{E} \mathbf{1}^{n}}=\left[\left(\mathbf{1} \wedge_{E} \mathbf{1}^{n}\right) \otimes p_{\mathbf{1}^{n}}^{\mathbf{1} \wedge_{E} \mathbf{1}^{n}}\right] \circ \Delta_{\mathbf{1} \wedge_{E} \mathbf{1}^{n}}
$$

and $l^{-1} \frac{1 \wedge_{E} G}{G}={ }^{1} \rho_{\frac{1 \wedge_{E} G}{G}}$ so that

$$
\left(i_{\mathbf{1}^{1}}^{\mathbf{1}^{n+1}} \otimes \frac{\mathbf{1}^{n+1}}{\mathbf{1}^{n}}\right) \circ l^{-1} \underset{\frac{1 \wedge_{G} G}{G}}{ } \circ p_{\mathbf{1}^{n}}^{\mathbf{1}^{n+1}}=\left(\mathbf{1}^{n+1} \otimes p_{\mathbf{1}^{n}}^{\mathbf{1}^{n+1}}\right) \circ \Delta_{\mathbf{1}^{n+1}} .
$$

We compute

$$
\begin{aligned}
& \left(i_{\mathbf{1}^{n+1}}^{E} \otimes \frac{i_{\mathbf{1}^{n+1}}^{E}}{\mathbf{1}^{n}}\right) \circ\left(\mathbf{1}^{n+1} \otimes p_{\mathbf{1}^{n+1}}^{\mathbf{1}^{n+1}}\right) \circ \alpha_{\mathbf{1}^{n+1}} \\
& =\left(i_{\mathbf{1}^{n+1}}^{E} \otimes \frac{i_{\mathbf{1}^{n+1}}^{E}}{\mathbf{1}^{n}}\right) \circ\left[\begin{array}{c}
\left(\mathbf{1}^{n+1} \otimes p_{\mathbf{1}^{n}}^{\mathbf{1}^{n+1}} i_{\mathbf{1}^{1}}^{\mathbf{1}^{n}}\right) \circ r_{\mathbf{1}^{n+1}}^{-1}+\left(i_{\mathbf{1}^{1}}^{\mathbf{1}^{n+1}} \otimes p_{\mathbf{1}^{n}}^{\mathbf{1}^{n+1}}\right) \circ l_{\mathbf{1}^{n+1}}^{-1}+ \\
-\left(\mathbf{1}^{n+1} \otimes p_{\mathbf{1}^{n}}^{\mathbf{1}^{n+1}}\right) \circ \Delta_{\mathbf{1}^{n+1}}
\end{array}\right] \\
& =\left(i_{\mathbf{1}^{n+1}}^{E} \otimes \frac{i_{\mathbf{1}^{n+1}}^{E}}{\mathbf{1}^{n}}\right) \circ\left[\left(i_{\mathbf{1}^{1}}^{\mathbf{1}^{n+1}} \otimes p_{\mathbf{1}^{n}}^{\mathbf{1}^{n+1}}\right) \circ l_{\mathbf{1}^{n+1}}^{-1}-\left(\mathbf{1}^{n+1} \otimes p_{\mathbf{1}^{n}}^{\mathbf{1}^{n+1}}\right) \circ \Delta_{\mathbf{1}^{n+1}}\right]
\end{aligned}
$$

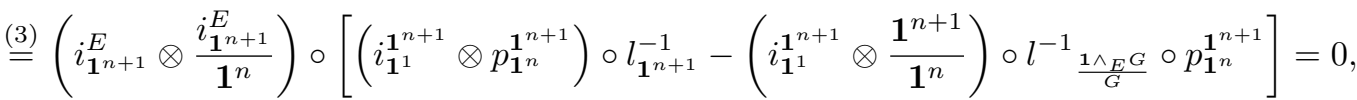

where the last equality follows by naturality of the unit constraint.

Since $i_{\mathbf{1}^{n+1}}^{E} \otimes \frac{i_{1^{n+1}}^{E}}{\mathbf{1}^{n}}$ is a monomorphism, we obtain

$$
\left(\mathbf{1}^{n+1} \otimes p_{\mathbf{1}^{n}}^{\mathbf{1}^{n+1}}\right) \circ \alpha_{\mathbf{1}^{n+1}}=0
$$

so that, as the above sequence is exact, by the universal property of kernels, there exists a unique morphism $\beta_{n}: \mathbf{1}^{n+1} \rightarrow \mathbf{1}^{n+1} \otimes \mathbf{1}^{n}$ such that

$$
\left(\mathbf{1}^{n+1} \otimes i_{\mathbf{1}^{n}}^{\mathbf{1}^{n+1}}\right) \circ \beta_{n}=\alpha_{\mathbf{1}^{n+1}} .
$$


By applying the functor $(-) \otimes \mathbf{1}^{n}$ to $(\boldsymbol{\nabla})$, we get

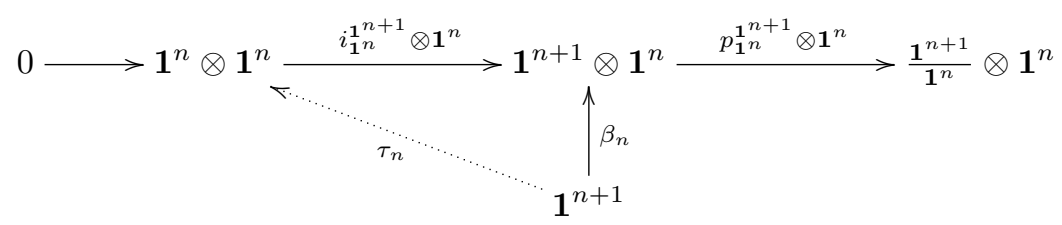

We have

$$
\begin{aligned}
&\left(\frac{\mathbf{1}^{n+1}}{\mathbf{1}^{n}} \otimes i_{\mathbf{1}^{n}}^{\mathbf{1}^{n+1}}\right) \circ\left(p_{\mathbf{1}^{n}}^{\mathbf{1}^{n+1}} \otimes \mathbf{1}^{n}\right) \circ \beta_{n}=\left(p_{\mathbf{1}^{n}}^{\mathbf{1}^{n+1}} \otimes \mathbf{1}^{n+1}\right) \circ\left(\mathbf{1}^{n+1} \otimes i_{\mathbf{1}^{n}}^{\mathbf{1}^{n+1}}\right) \circ \beta_{n} \\
& \stackrel{(\text { 回) }}{=}\left(p_{\mathbf{1}^{n}}^{\mathbf{1}^{n+1}} \otimes \mathbf{1}^{n+1}\right) \circ \alpha_{\mathbf{1}^{n+1}}=0
\end{aligned}
$$

where the last equality can be proved similarly to $(\mathbb{\Psi})$. Since $\frac{\mathbf{1}^{n+1}}{\mathbf{1}^{n}} \otimes i_{\mathbf{1}^{n}}^{\mathbf{1}^{n+1}}$ is a monomorphism we get $\left(p_{\mathbf{1}^{n}}^{\mathbf{1}^{n+1}} \otimes \mathbf{1}^{n}\right) \circ \beta_{n}=0$ so that, as the previous sequence is exact, by the universal property of kernels there exists a unique morphism $\tau_{n}: \mathbf{1}^{n+1} \rightarrow \mathbf{1}^{n} \otimes \mathbf{1}^{n}$ such that $\left(i_{\mathbf{1}^{n}}^{n+1} \otimes \mathbf{1}^{n}\right) \circ \tau_{n}=\beta_{n}$. Finally we have

$$
\left(i_{\mathbf{1}^{n}}^{\mathbf{1}^{n+1}} \otimes i_{\mathbf{1}^{n}}^{\mathbf{1}^{n+1}}\right) \circ \tau_{n}=\left(\mathbf{1}^{n+1} \otimes i_{\mathbf{1}^{n}}^{\mathbf{1}^{n+1}}\right) \circ\left(i_{\mathbf{1}^{n}}^{\mathbf{n}^{n+1}} \otimes \mathbf{1}^{n}\right) \circ \tau_{n}=\left(\mathbf{1}^{n+1} \otimes i_{\mathbf{1}^{n}}^{\mathbf{1}^{n+1}}\right) \circ \beta_{n}=\alpha_{\mathbf{1}^{n+1}} .
$$

THEOREM 2.8. Let $\left(\left(E, \Delta_{E}, \varepsilon_{E}\right), u_{E}=i_{\mathbf{1}}^{E}\right)$ be a coaugmented coalgebra in a coabelian monoidal category $\mathcal{M}$. Then $\varepsilon_{E} \circ i_{P(E)}=0$ and

$$
\left(\mathbf{1} \wedge_{\mathbf{E}} \mathbf{1}=\mathbf{1}^{\wedge_{E}^{2}}, i_{\mathbf{1}^{\wedge}{ }_{E}^{2}}\right)=\left(\mathbf{1} \oplus P(E), \nabla\left(u_{E}, i_{P(E)}\right)\right),
$$

where $\nabla\left(u_{E}, i_{P(E)}\right): \mathbf{1} \oplus P(E) \rightarrow E$ denotes the codiagonal morphism associated to $u_{E}$ and $i_{P(E)}$.

Proof. Set $P=P(E)$. Since $\left(E, u_{E}\right)$ is a coaugmented coalgebra, we apply Lemma 2.0 coalgebra homomorphism $\varepsilon_{E}: E \rightarrow \mathbf{1}$. Thus $\left(\mathbf{1}, u_{\mathbf{1}}=\varepsilon_{E} i_{\mathbf{1}}^{E}=\operatorname{Id}_{\mathbf{1}}\right)$ is a coaugmented coalgebra and $\alpha_{1} \circ \varepsilon_{E}=\left(\varepsilon_{E} \otimes \varepsilon_{E}\right) \circ \alpha_{E}$. We have

$$
\alpha_{1} \circ \varepsilon_{E} \circ i_{P}=\left(\varepsilon_{E} \otimes \varepsilon_{E}\right) \circ \alpha_{E} \circ i_{P}=0 .
$$

By definition, we have

$$
\alpha_{1}=\left(\mathbf{1} \otimes u_{1}\right) \circ r_{1}^{-1}+\left(u_{1} \otimes \mathbf{1}\right) \circ l_{1}^{-1}-\Delta_{1}=r_{1}^{-1}+l_{1}^{-1}-l_{1}^{-1}=r_{1}^{-1} .
$$

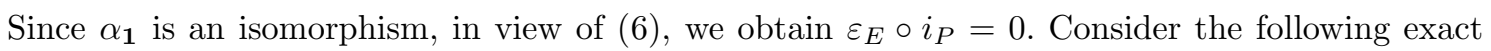
sequence

$$
0 \rightarrow \mathbf{1} \stackrel{i_{1}^{E}}{\longrightarrow} E \stackrel{p_{1}^{E}}{\longrightarrow} \frac{E}{\mathbf{1}} \rightarrow 0
$$

Since $\varepsilon_{E} \circ i_{\mathbf{1}}^{E}=\operatorname{Id}_{\mathbf{1}}$, there exists a unique morphism $a: \frac{E}{\mathbf{1}} \rightarrow E$ such that $\operatorname{Id}_{E}=i_{\mathbf{1}}^{E} \varepsilon_{E}+a p_{\mathbf{1}}^{E}$. Clearly the following sequence

$$
0 \rightarrow \frac{E}{\mathbf{1}} \stackrel{a}{\longrightarrow} E \stackrel{\varepsilon_{E}}{\longrightarrow} \mathbf{1} \rightarrow 0
$$

is exact. From $\varepsilon_{E} \circ i_{P}=0$, we get that there exists a unique morphism $i_{P}^{\prime}: P \rightarrow \frac{E}{\mathbf{1}}$ such that $a \circ i_{P}^{\prime}=i_{P}$. Thus

$$
\nabla\left(i_{\mathbf{1}}^{E}, a\right) \circ\left(\operatorname{Id}_{\mathbf{1}} \oplus i_{P}^{\prime}\right)=\nabla\left(i_{\mathbf{1}}^{E} \circ \operatorname{Id}_{\mathbf{1}}, a \circ i_{P}^{\prime}\right)=\nabla\left(i_{\mathbf{1}}^{E}, i_{P}\right) .
$$

where $\nabla\left(i_{\mathbf{1}}^{E}, a\right): \mathbf{1} \oplus \frac{E}{\mathbf{1}} \rightarrow E$ is the codiagonal morphism associated to $i_{\mathbf{1}}^{E}$ and $a$. Since $\nabla\left(i_{\mathbf{1}}^{E}, a\right)$ is an isomorphism and $\operatorname{Id}_{\mathbf{1}} \oplus i_{P}^{\prime}$ is a monomorphism, we get that $\nabla\left(i_{\mathbf{1}}^{E}, i_{P}\right)$ is a monomorphism. Let us prove that

$$
\alpha_{E} \circ\left(\underset{\mathbf{1}^{\wedge} E}{i_{E}^{E}}-i_{\mathbf{1}}^{E} \circ \varepsilon_{E} \circ i_{\mathbf{1}_{E}^{\wedge}}^{E}\right)=0 .
$$


By Lemma 2.5 and Lemma [2.7, we have

$$
\alpha_{E} \circ i_{\mathbf{1}^{\wedge} E}^{E}=\left(\underset{\mathbf{1}^{\wedge} E}{i_{E}^{E}} \otimes i_{\mathbf{1}^{\wedge} E}^{E}\right) \circ \alpha_{\mathbf{1}^{2}}=\left(i_{\mathbf{1}^{\wedge} E}^{E} \otimes i_{\mathbf{1}^{\wedge} E}^{E}\right) \circ\left(i_{\mathbf{1}}^{\mathbf{1}^{\wedge}{ }_{E}^{2}} \otimes i_{\mathbf{1}}^{\mathbf{1}^{\wedge}{ }_{E}^{2}}\right) \circ \tau_{1}=\left(i_{\mathbf{1}}^{E} \otimes i_{\mathbf{1}}^{E}\right) \circ \tau_{1}
$$

for a suitable $\tau_{1}: \mathbf{1}^{\wedge_{E}^{2}} \rightarrow \mathbf{1} \otimes \mathbf{1}$. Then, by Lemma $[\mathbf{L}$, we have

$$
\left(\varepsilon_{E} \otimes \varepsilon_{E}\right) \circ \alpha_{E}=\alpha_{1} \circ \varepsilon_{E} \stackrel{(\mathbb{E})}{=} r_{\mathbf{1}}^{-1} \circ \varepsilon_{E} .
$$

so that

$$
\tau_{1}=\left(\varepsilon_{E} \otimes \varepsilon_{E}\right) \circ\left(i_{\mathbf{1}}^{E} \otimes i_{\mathbf{1}}^{E}\right) \circ \tau_{1}=\left(\varepsilon_{E} \otimes \varepsilon_{E}\right) \circ \alpha_{E} \circ i_{\mathbf{1}^{\wedge} \wedge_{E}^{2}}^{\stackrel{(\mathbb{\nabla})}{=}} r_{\mathbf{1}}^{-1} \circ \varepsilon_{E} \circ i_{\mathbf{1}^{\wedge} E}^{E} \cdot
$$

Then

$$
\alpha_{E} \circ i_{\mathbf{1}^{\wedge} E}^{E}=\left(i_{\mathbf{1}}^{E} \otimes i_{\mathbf{1}}^{E}\right) \circ \tau_{1}=\left(i_{\mathbf{1}}^{E} \otimes i_{\mathbf{1}}^{E}\right) \circ r_{\mathbf{1}}^{-1} \circ \varepsilon_{E} \circ i_{\mathbf{1}^{\wedge}{ }_{E}^{2}}^{i^{2}} .
$$

On the other, by Lemma $\boldsymbol{L . 5}$, hand we have

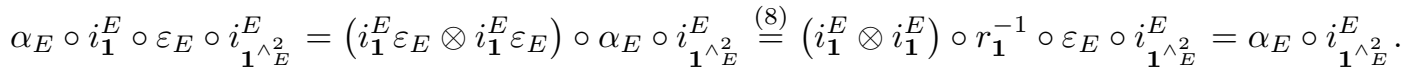

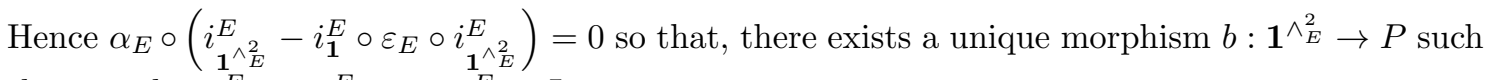
that $i_{P} \circ b=i_{\mathbf{1}^{\wedge}{ }_{E}^{2}}^{{ }^{\wedge}}-i_{\mathbf{1}}^{E} \circ \varepsilon_{E} \circ i_{\mathbf{1}_{E}^{\wedge}}^{i^{2}}$. Let

$$
\Delta\left(\varepsilon_{E} i_{\mathbf{1}^{\wedge}{ }_{E}^{2}}^{E}, b\right): \mathbf{1}^{\wedge}{ }_{E}^{2} \rightarrow \mathbf{1} \oplus P
$$

be the diagonal morphism of $\varepsilon_{E} i_{\mathbf{1}^{\wedge}{ }_{E}^{E}}^{{ }^{2}}: \mathbf{1}^{\wedge_{E}^{2}} \rightarrow \mathbf{1}$ and $b: \mathbf{1}^{\wedge_{E}^{2}} \rightarrow P$. We have

$$
\nabla\left(i_{\mathbf{1}}^{E}, i_{P}\right) \circ \Delta\left(\varepsilon_{E} i_{\mathbf{1}^{\wedge} E}^{E}, b\right)=i_{\mathbf{1}}^{E} \circ \varepsilon_{E} \circ i_{\mathbf{1}^{\wedge} E}^{E}+i_{P} \circ b=i_{\mathbf{1}^{\wedge}{ }_{E}^{2}}^{i^{2}}
$$

so that

$$
\nabla\left(i_{\mathbf{1}}^{E}, i_{P}\right) \circ \Delta\left(\varepsilon i_{\mathbf{1}^{\wedge} E}^{E}, b\right)=i_{\mathbf{1}^{\wedge}{ }_{E}^{2}}^{E} .
$$

We have

$$
\begin{aligned}
& \left(p_{\mathbf{1}}^{E} \otimes p_{\mathbf{1}}^{E}\right) \circ \Delta_{E} \circ \nabla\left(i_{\mathbf{1}}^{E}, i_{P}\right) \\
= & \nabla\left[\left(p_{\mathbf{1}}^{E} \otimes p_{\mathbf{1}}^{E}\right) \circ \Delta_{E} \circ i_{\mathbf{1}}^{E},\left(p_{\mathbf{1}}^{E} \otimes p_{\mathbf{1}}^{E}\right) \circ \Delta_{E} \circ i_{P}\right] \\
= & \nabla\left\{\left(p_{\mathbf{1}}^{E} \otimes p_{\mathbf{1}}^{E}\right) \circ\left(i_{\mathbf{1}}^{E} \otimes i_{\mathbf{1}}^{E}\right) \circ \Delta_{E},\left(p_{\mathbf{1}}^{E} \otimes p_{\mathbf{1}}^{E}\right) \circ\left[\left(E \otimes i_{\mathbf{1}}^{E}\right) \circ r_{E}^{-1}+\left(i_{\mathbf{1}}^{E} \otimes E\right) \circ l_{E}^{-1}\right]\right\}=0
\end{aligned}
$$

so that there exists a unique morphism $\Gamma\left(i_{\mathbf{1}}^{E}, i_{P}\right): \mathbf{1} \oplus P \rightarrow \mathbf{1}^{\wedge_{E}^{2}}$ such that

$$
\nabla\left(i_{\mathbf{1}}^{E}, i_{P}\right)=i_{\mathbf{1}^{\wedge}{ }_{E}^{E}}^{E} \circ \Gamma\left(i_{\mathbf{1}}^{E}, i_{P}\right) .
$$

Since $\nabla\left(i_{\mathbf{1}}^{E}, i_{P}\right)$ is a monomorphism, so is $\Gamma\left(i_{\mathbf{1}}^{E}, i_{P}\right)$. On the other hand, we get

$$
i_{\mathbf{1}^{\wedge}{ }_{E}^{2}}^{E} \circ \Gamma\left(i_{\mathbf{1}}^{E}, i_{P}\right) \circ \Delta\left(\varepsilon i_{\mathbf{1}_{\text {^E }}^{E}}^{E}, b\right) \stackrel{(\mathbb{Q})}{=} i_{\mathbf{1}^{\wedge}{ }_{E}^{E}}^{E} .
$$

Since $i_{\mathbf{1}_{E}^{\wedge}}^{E}$ is a monomorphism, we get $\Gamma\left(i_{\mathbf{1}}^{E}, i_{P}\right) \circ \Delta\left(\varepsilon i_{\mathbf{1}_{E}^{(2}}^{i_{E}^{2}}, b\right)=\operatorname{Id}_{\mathbf{1}^{\wedge}{ }_{E}^{2}}$ and hence $\Gamma\left(i_{\mathbf{1}}^{E}, i_{P}\right)$ is also an epimorphism. Thus $\Gamma\left(i_{\mathbf{1}}^{E}, i_{P}\right)$ and $\Delta\left(\varepsilon i_{\mathbf{1}^{\wedge}{ }_{E}^{2}}^{2}, b\right)$ are mutual inverses.

Definition 2.9. A graded coalgebra $\left(C=\oplus_{n \in \mathbb{N}} C_{n}, \Delta_{C}, \varepsilon_{C}\right)$ in a cocomplete monoidal category $\mathcal{M}$ is called 0-connected whenever $\varepsilon_{0}^{C}=\varepsilon_{C} i_{0}^{C}: C_{0} \rightarrow \mathbf{1}$ is an isomorphism

A graded coalgebra $C$ in a cocomplete monoidal category $\mathcal{M}$ is called strictly graded whenever

1) $C$ is 0-connected;

2) $C$ is a strongly $\mathbb{N}$-graded coalgebra.

Next theorem provides our main example of a strictly graded coalgebra. 
THEOREM 2.10. Let $\mathcal{M}$ be a cocomplete coabelian monoidal category such that the tensor product commutes with direct sums. Let $\left((C, \Delta, \varepsilon), u_{C}\right)$ be a coaugmented coalgebra in $\mathcal{M}$.

Then the associated graded coalgebra

$$
g r_{1} C=\mathbf{1} \oplus \frac{\mathbf{1} \wedge_{C} \mathbf{1}}{\mathbf{1}} \oplus \frac{\mathbf{1} \wedge_{C} \mathbf{1} \wedge_{C} \mathbf{1}}{\mathbf{1} \wedge_{C} \mathbf{1}} \oplus \cdots
$$

is a strictly graded coalgebra.

Proof. By [A]2, Theorem 2.10], we have that $\left(g r_{1} C, \Delta_{g r_{1} C}, \varepsilon_{g r_{1} C}=\varepsilon_{C} \circ u_{C} \circ p_{0}^{g r_{1} C}\right)$ is a strongly $\mathbb{N}$-graded coalgebra. Since $u_{C}$ is a coalgebra homomorphism, we get

$$
\varepsilon_{g r_{1} C}=\varepsilon \circ u_{C} \circ p_{0}^{g r_{1} C}=p_{0}^{g r_{1} C} .
$$

It is now clear that $\varepsilon_{0}^{g r_{1} C}:=\varepsilon_{g r_{1} C} \circ i_{0}^{g r_{1} C}=\operatorname{Id}_{1}$ so that $g r_{1} C$ also 0 -connected and hence it is a strictly graded coalgebra.

TheOREM 2.11. Let $\left(C=\oplus_{n \in \mathbb{N}} C_{n}, \Delta_{C}, \varepsilon_{C}\right)$ be a 0 -connected graded coalgebra in a cocomplete coabelian monoidal category $\mathcal{M}$ (e.g. $C$ is strictly graded). Then

1) $\left(\left(C, \Delta_{C}, \varepsilon_{C}\right), u_{C}=i_{\mathbf{1}}^{C}\right)$ is a connected coalgebra where $u_{C}:=i_{0}^{C} \varepsilon_{0}^{-1}: \mathbf{1} \rightarrow C$.

2) $\left(C_{0}^{\wedge{ }_{C}^{2}}, i_{C_{0}^{\wedge}{ }_{C}^{2}}\right)=\left(C_{0} \oplus P(C), \nabla\left(i_{0}^{C}, i_{P(C)}\right)\right)$, where $\nabla\left(i_{0}^{C}, i_{P(C)}\right): C_{0} \oplus P(C) \rightarrow C$ denotes the diagonal morphism associated to $i_{0}^{C}$ and $i_{P(C)}$.

Moreover if $\mathcal{M}$ is also complete and satisfies AB5, then the following assertions are equivalent:

(a) $C$ is a strongly $\mathbb{N}$-graded coalgebra.

(b) $\left(C_{1}, i_{1}^{C}\right)=\left(P(C), i_{P(C)}\right)$.

In particular, when $(b)$ holds, $C$ is a strictly graded coalgebra.

Proof. 1) By Proposition [AM], Proposition 2.5], $\left(C_{0}, \Delta_{0}=\Delta_{0,0}, \varepsilon_{0}=\varepsilon i_{0}^{C}\right)$ is a coalgebra in $\mathcal{M}$ and $i_{0}^{C}$ is a coalgebra homomorphism. Hence $\varepsilon_{0}$ and $i_{0}^{C}$ are both coalgebra homomorphisms so that $\delta:=i_{0}^{C} \varepsilon_{0}^{-1}$ is a coalgebra homomorphism and hence $\left(\left(C, \Delta_{C}, \varepsilon_{C}\right), \delta\right)$ is a coaugmented coalgebra. By [AMS], Proposition 3.3], we have $C=\underline{\lim }\left(C_{0}^{\wedge{ }_{C}^{t}}\right)_{t \in \mathbb{N}}$. Since $\varepsilon_{0}$ is a coalgebra isomorphism, we conclude that $\lim _{\longrightarrow}\left(\mathbf{1}_{C}^{n}\right)_{n \in \mathbb{N}}=C$ i.e. that $\left(\left(C, \Delta_{C}, \varepsilon_{C}\right), \delta\right)$ is a connected coalgebra.

2) It follows by 1 ) and in view of Theorem 2.8 .

Now, assume that $\mathcal{M}$ is also complete and satisfies $A B 5$ and let us prove that $(a)$ and $(b)$ are equivalent. By 2), we have

$$
\left(C_{0}^{\wedge_{C}^{2}}, \delta_{2}\right)=\left(C_{0} \oplus P(C), \nabla\left(i_{0}^{C}, i_{P(C)}\right)\right) .
$$

Then, by [AM], Theorem 2.22], (a) is equivalent to

and hence to

$$
\left(C_{0} \oplus C_{1}, \nabla\left(i_{0}^{C}, i_{1}^{C}\right)\right)=\left(C_{0}^{\wedge_{C}^{2}}, \delta_{2}\right)
$$

$$
\left(C_{0} \oplus C_{1}, \nabla\left(i_{0}^{C}, i_{1}^{C}\right)\right)=\left(C_{0} \oplus P(C), \nabla\left(i_{0}^{C}, i_{P(C)}\right)\right) .
$$

$(b) \Rightarrow$ (प्]) It is trivial.

(ㅁ] $) \Rightarrow(b)$ By hypothesis there exists an isomorphism $\Lambda: C_{0} \oplus P(C) \rightarrow C_{0} \oplus C_{1}$ such that $\nabla\left(i_{0}^{C}, i_{P(C)}^{C}\right)=\nabla\left(i_{0}^{C}, i_{1}^{C}\right) \circ \Lambda$.

Let $\pi_{C_{a}}^{C_{0} \oplus C_{1}}: C_{0} \oplus C_{1} \rightarrow C_{a}$ be the canonical projection for $a=0,1$. We have

$$
\varepsilon_{C} \circ \nabla\left(i_{0}^{C}, i_{1}^{C}\right)=\nabla\left(\varepsilon_{C} \circ i_{0}^{C}, \varepsilon_{C} \circ i_{1}^{C}\right)=\nabla\left(\varepsilon_{0}, 0_{H o m\left(C_{1}, \mathbf{1}\right)}\right)=\varepsilon_{0} \circ \pi_{C_{0}}^{C_{0} \oplus C_{1}}
$$

and by Theorem [2.8, we have

$$
\varepsilon_{C} \circ \nabla\left(i_{0}^{C}, i_{P(C)}\right)=\nabla\left(\varepsilon_{C} \circ i_{0}^{C}, \varepsilon_{C} \circ i_{P(C)}^{C}\right)=\nabla\left(\varepsilon_{0}, 0_{H o m(P(C), \mathbf{1})}\right)=\varepsilon_{0} \circ \pi_{C_{0}}^{C_{0} \oplus P(C)} .
$$

Hence, by definition of $\Lambda$ we get

$$
\varepsilon_{0} \circ \pi_{C_{0}}^{C_{0} \oplus C_{1}} \circ \Lambda=\varepsilon_{C} \circ \nabla\left(i_{0}^{C}, i_{1}^{C}\right) \circ \Lambda=\varepsilon_{C} \circ \nabla\left(i_{0}^{C}, i_{P(C)}\right)=\varepsilon_{0} \circ \pi_{C_{0}}^{C_{0} \oplus P(C)} .
$$


Since $\varepsilon_{0}$ is an isomorphism, we get that

$$
\pi_{C_{0}}^{C_{0} \oplus C_{1}} \circ \Lambda=\pi_{C_{0}}^{C_{0} \oplus P(C)} .
$$

Consider the following diagram

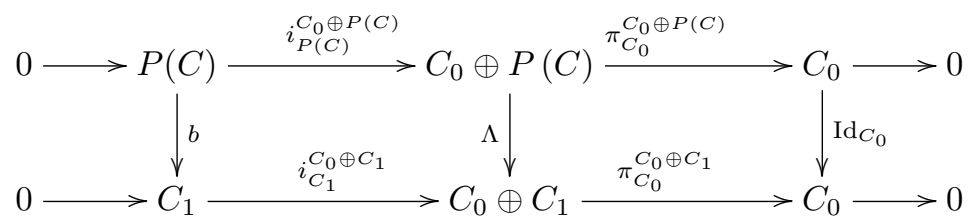

where the rows are exact and the right square commutes. Hence there is a unique morphism $b: P(C) \rightarrow C_{1}$ such that the left square commutes too. Clearly $b$ is an isomorphism. Moreover

$$
i_{1}^{C} \circ b=\nabla\left(i_{0}^{C}, i_{1}^{C}\right) \circ i_{C_{1}}^{C_{0} \oplus C_{1}} \circ b=\nabla\left(i_{0}^{C}, i_{1}^{C}\right) \circ \Lambda \circ i_{P(C)}^{C_{0} \oplus P(C)}=\nabla\left(i_{0}^{C}, i_{P(C)}^{C}\right) \circ i_{P(C)}^{C_{0} \oplus P(C)}=i_{P(C)}^{C}
$$

so that $\left(C_{1}, i_{1}^{C}\right)=\left(P(C), i_{P(C)}^{C}\right)$.

Remark 2.12. Let $\left(C=\oplus_{n \in \mathbb{N}} C_{n}, \Delta_{C}, \varepsilon_{C}\right)$ be a graded coalgebra in a cocomplete and complete coabelian monoidal category $\mathcal{M}$ satisfying $A B 5$. In view of Theorem $2 . \mathbb{}, C$ is strictly graded if and only if it is 0-connected and

$$
\left(C_{1}, i_{1}^{C}\right)=\left(P(C), i_{P(C)}\right) .
$$

Note that, when $\mathcal{M}$ is the category of vector spaces over a field $K$, our definition agrees with Sweedler's one in $[\mathbf{5 w}$, page 232].

THEOREM 2.13. Let $\left(B, m_{B}, u_{B}, \Delta_{B}, \varepsilon_{B}\right)$ be a braided graded bialgebra in a cocomplete and complete braided monoidal category $(\mathcal{M}, c)$ such that $\mathcal{M}$ is abelian satisfying AB5. Assume that the tensor products are additive, commute with direct sums and are (two-sided) exact. Assume that $B$ is 0 -connected as a coalgebra.

Then $\left(\left(B, \Delta_{B}, \varepsilon_{B}\right), u_{B}\right)$ is a connected coalgebra.

Moreover $B$ is the braided bialgebra of type one $B_{0}\left[B_{1}\right]$ associated to $B_{0}$ and $B_{1}$ if and only if

$$
\left(\oplus_{n \geq 1} B_{n}\right)^{2}=\oplus_{n \geq 2} B_{n} \quad \text { and } \quad P(B)=B_{1} .
$$

Proof. By [AM], Theorem 6.10] and Theorem [س], $\left(\left(B, \Delta_{B}, \varepsilon_{B}\right), \delta\right)$ is a connected coalgebra where $\delta:=i_{0}^{B} \varepsilon_{0}^{-1}: \mathbf{1} \rightarrow B$. Moreover $B$ is the braided bialgebra of type one $B_{0}\left[B_{1}\right]$ associated to $B_{0}$ and $B_{1}$ if and only if $\left(\oplus_{n \geq 1} B_{n}\right)^{2}=\oplus_{n \geq 2} B_{n}$ and $P(B)=B_{1}$.

Let us prove that $\bar{\delta}=u_{B}$.

By [AMD, Propositions 2.5 and 3.4], $\varepsilon_{B}=\varepsilon_{0} \circ p_{0}^{B}$ and $u_{B}=i_{0}^{B} \circ u_{0}$ where $u_{0}=p_{0}^{B} \circ u_{B}$. Thus

$$
\mathrm{Id}_{\mathbf{1}}=\varepsilon_{B} \circ u_{B}=\varepsilon_{0} \circ p_{0}^{B} \circ i_{0}^{B} \circ u_{0}=\varepsilon_{0} \circ u_{0}
$$

and hence $u_{0}=\varepsilon_{0}^{-1}$. Then we get $u_{B}=i_{0}^{B} \circ u_{0}=i_{0}^{B} \circ \varepsilon_{0}^{-1}$.

REMARK 2.14. Recall that a TOBA (also called braided Hopf algebra of type one) WG, Definition 3.2.3] in the category ${ }_{H}^{H} \mathcal{Y D}$ of Yetter Drinfeld modules over an ordinary $K$-Hopf algebra $H$ is a graded bialgebra $T=\oplus_{n \in \mathbb{N}} T_{n}$ in this category such that

$$
T_{0} \simeq K, \quad\left(\oplus_{n \geq 1} T_{n}\right)^{2}=\oplus_{n \geq 2} T_{n}, \quad \text { and } \quad P(T)=T_{1} .
$$

Therefore TOBAs are exactly the bialgebras described in Theorem [.] 3 in the case when $\mathcal{M}=$ ${ }_{H}^{H} \mathcal{Y} \mathcal{D}$. 


\section{REFERENCES}

[AM1] A. Ardizzoni and C. Menini, Braided Bialgebras of Type One, Comm. Algebra, to appear.

[AM2] A. Ardizzoni and C. Menini, Braided Bialgebras of Type One: Applications, submitted. (arXiv:0704.2106v1)

[AG] N. Andruskiewitsch and M. Graña, Braided Hopf algebras over non-abelian finite groups, Colloquium on Operator Algebras and Quantum Groups (Spanish) (Vaquerías, 1997). Bol. Acad. Nac. Cienc. (Córdoba) 63 (1999), 45-78.

[Ar] A. Ardizzoni, Wedge Products and Cotensor Coalgebras in Monoidal Categories, Algebr. Represent. Theory, to appear.

[AMS1] A. Ardizzoni, C. Menini and D. Stefan, Cotensor Coalgebras in Monoidal Categories, Comm. Algebra, Vol. 35, N. 1 (2007), 25-70.

[AMS2] A. Ardizzoni, C. Menini and D. Stefan, Hochschild Cohomology And 'Smoothness' In Monoidal Categories, J. Pure Appl. Algebra, 208 (2007), 297-330.

KKa] Kassel, Quantum Groups, Graduate Text in Mathematics 155, Springer, 1995.

[Mj1] S. Majid, Foundations of quantum group theory, Cambridge University Press, 1995.

[NT] C. Năstăsescu, B. Torrecillas, Graded coalgebras, Tsukuba J. Math. 17 (1993), 461-479.

[Ni] Nichols, W. D. Bialgebras of type one. Comm. Algebra 6 (1978), no. 15, 1521-1552.

[Sw] M. Sweedler, Hopf Algebras, Benjamin, New York, 1969.

University of Ferrara, Department of Mathematics, Via Machiavelli 35, Ferrara, I-44100, Italy

E-mail address: rdzlsn@unife.it

URL: http://www.unife.it/utenti/alessandro.ardizzoni

University of Ferrara, Department of Mathematics, Via Machiavelli 35, Ferrara, I-44100, Italy

E-mail address: men@unife.it

$U R L:$ http://www.unife.it/utenti/claudia.menini 